\title{
Pain and Tension Are Reduced Among Hospital Nurses After On-Site Massage Treatments: A Pilot Study
}

\author{
JOEL KATZ, PhD \\ ADAROSE WOWK, BSc, RN \\ DIANNE CULP, RMT \\ HEATHER WAKELING
}

\begin{abstract}
Tension and pain are common occupational hazards of modern-day nursing, especially given recent changes to the health care system. The aims of the pilot study were (1) to evaluate the feasibility of carrying out a series of eight 15-minute workplace-based massage treatments, and (2) to determine whether massage therapy reduced pain and stress experienced by nursing staff at a large teaching hospital. Twelve hospital staff (10 registered nurses and 2 nonmedical ward staff) working in a large tertiary care center volunteered to participate. Participants received up to eight, workplace-based, 15-minute Swedish massage treatments provided by registered massage therapists. Pain, tension, relaxation, and the Profile of Mood States were measured before and after each massage session. Pain intensity and tension levels were significantly lower after massage $(P<.01)$. In addition, relaxation levels and overall mood state improved significantly after treatments $(P<.01)$. The results of this pilot study support the feasibility of an eight-session, workplacebased, massage therapy program for pain and tension experienced by nurses working in a large teaching hospital. Further research is warranted to study the efficacy of workplace massage in reducing stress and improving overall mood.
\end{abstract}

Joel Katz, PhD, is Psychologist-in-Chief of the Department of Psychology, The Toronto Hospital, Associate Director of the Acute Pain Research Unit, The Toronto Hospital and Mount Sinai Hospital, and Associate Professor in the Department of Public Health Sciences and Department of Anaesthesia, University of Toronto, Toronto, Canada; Adarose Wowk, BSc, RN, is Research Coordinator in the Acute Pain Research Unit, Department of Anaesthesia, The Toronto Hospital and Mount Sinai Hospital, Toronto, Canada; Dianne Culp, RMT, is a Massage
Therapist at the Toronto Hospital Fitness Centre, Toronto, Canada; and Heather Wakeling is a Coordinator at The Toronto Hospital Fitness Centre, Toronto, Canada.

Address correspondence to Joel Katz, PhD, Department of Psychology, The Toronto Hospital, 200 Elizabeth St, CW 2-332, Toronto, ON, Canada M5G 2 C4. 
$\mathbf{T}$

HE GROWING recognition of the changing work environment and the negative impact of job stress on health ${ }^{1-3}$ have led to efforts to reduce workplace stress ${ }^{4-7}$ and to facilitate coping and a sense of control. ${ }^{5}$ Massage therapy is being used with increasing frequency to treat pain, stress, and burn-out in the general population ${ }^{8,9}$ and among health care professionals. ${ }^{4-6,10,11}$ Recent randomized, controlled studies have shown massage therapy to be effective in reducing a variety of negative mood states including depression, anxiety, fatigue, and confusion. ${ }^{5,10,12}$

It is possible that massage therapy would be of benefit to counter the stress experienced by nurses working in a hospital setting. However, because of long working hours and time constraints owing to shift work, massage therapy is not a feasible option unless it can be worksite-based. We are not aware of any studies that have assessed the feasibility or efficacy of on-site massage therapy for pain and stress reduction in nurses working in a large teaching hospital. The aims of the present pilot study were (1) to evaluate the feasibility of carrying out a series of eight 15-minute workplace-based massage treatments, and (2) to determine whether massage therapy reduced pain and stress experienced by nursing staff at a large teaching hospital. The results of this pilot study will be used to design a randomized, controlled trial of massage therapy to determine its efficacy.

\section{MATERIALS AND METHODS}

Approval to carry out the study was obtained from The Toronto Hospital Committee for Research on Human Subjects. All participants gave their informed, written consent to participate before entering the study.

\section{Measures}

At the beginning of the first session, participants completed a brief questionnaire measuring demographic information. The following measures were obtained immediately before and after each treatment.

Pain, tension, headache, and relaxation. Participants completed a brief questionnaire that assessed the presence and location of pain and tension in the head, neck, or shoulders, as well as the presence of headache and degree of relaxation. The degree of pain, tension, and relaxation was assessed with a $10 \mathrm{~cm}$ visual analogue scale ${ }^{13}$ with endpoints that were labeled with appropriate descriptors (eg, for the pain visual analogue scale, "no pain" and "worst possible pain").

Profile of Mood States. The Profile of Mood States (POMS) is a self-administered inventory that assesses six current mood states (tension-anxiety, depression-dejection, anger-hostility, vigor-activity, fatigue-inertia, and confusion-bewilderment) by way of 65 adjectives rated on 5-point Likert scales. A total mood disturbance score can also be calculated that consists of a weighted score from each subscale. The POMS subscales have internal consistency coefficients that range from .84 to .95 and a fair degree of test-retest reliability. ${ }^{14}$

Duration of treatment effects. At the beginning of sessions two through eight, participants completed a 5-point forced-choice evaluation form that assessed the duration of pain relief, tension reduction, and relaxation that resulted from the previous session (eg, for pain relief, the 5-point scale consisted of the following: 0 , no pain to relieve; 1 , no pain relief; 2 , less than 1 hour of pain relief; 3 , more than 1 hour but less than 24 hours of pain relief; and 4, more than 24 hours of pain relief).

\section{Procedure}

Staff were recruited after meeting with a nurse manager who notified her nursing staff of the research project. They were informed that a research project involving workplace massage treatment was being performed in an attempt to deal with the high levels of stress experienced by hospital nurses, and were asked to sign up on a voluntary basis. The following were the exclusion criteria for the study: recent head, neck, or shoulder surgery; pins or joint fusion in the head or neck; a diagnosis of cancer, epilepsy, anemia, or diabetes; or a history of easy bruising, earaches, sinus infections, or vascular migraines.

Sessions took place in a common staff lounge. At the beginning of the first session, participants gave written voluntary consent, completed a brief demographic questionnaire, and completed pretreatment questionnaires (assessing pain, tension, degree of relaxation, and mood state). After the treatment, participants completed post-treatment questionnaires and the next massage session was scheduled. The procedure for all subsequent sessions followed the same format with the exception that participants 
also rated the duration of pain relief, tension relief, and relaxation obtained from the previous session.

Massage therapy. When the questionnaires had been completed, participants moved to a quieter corner of the room for the massage treatment. Participants were fully clothed and seated for the massage. The massage treatments were carried out by two professional registered massage therapists who used the Swedish massage technique. Each participant was assigned to one massage therapist for the duration of the study. The massage procedure consisted of (1) static compressions across the upper trapezius using the heel of the hands; (2) squeezing of the deltoids; (3) lifting the shoulder girdle from the lateral border of the scapulas; (4) thumb kneading of the interscapular area from the inferior angle of the scapula up to the neck; (5) opposing thumb compressions along the nuchal ligament from $\mathrm{C} 7$ to the inion; (6) circular thumb kneading on both sides of the spine from the occiput down to $\mathrm{C} 7$; (7) heel of the hand compression along the erectors down to the sacrum; (8) knuckle walking alternately from the sacrum up to $\mathrm{T} 1$; (9) thumb compression and kneading to the paraspinals from $\mathrm{T} 1$ down to the sacrum; (10) palmar compressions to the lateral border of the back (ie, latissimus dorsi) on both sides; (11) knuckle kneading of the infraspinous fossa; (12) static thumb compression along the spine of scapula both inferior and superior; (13) circular thumb kneading and ischemic compression to midtrapezius; (14) muscle squeezing to the anterior border of the upper trapezius using both hands, unilaterally; (15) ischemic pressure to scaleness; (16) repeating steps 14 and 15 on the other side; (17) fingertip circular kneading to the mastoid and medially to the nuchal ligament along the occiput of the skull, bilaterally; (18) compression of the web space of the hand (thenar eminance to first digit); (19) mobilizations to the wrists; (20) muscle squeezing of the forearm, wrist to the elbow, with circular thumb kneading over the common extensor tendon; (21) muscle squeezing of the upper arm from the elbow to the glenohumeral joint; and (22) specific thumb and forefinger squeezing applied to the anterior and posterior border of the deltoids.

\section{Data Reduction and Statistical Analyses}

To simplify data analysis and data presentation, pretreatment and post-treatment variables (pain,
Table 1. Demographic and Pretreatment Clinical Variables for the 12 Participants

\begin{tabular}{|c|c|}
\hline Gender (\% women) & 100 \\
\hline Does not exercise regularly (\%) & 8 \\
\hline Wears contact lenses $(\%)$ & 17 \\
\hline Has tension headaches (\%) & 75 \\
\hline Occupation (\% RNs) & 83 \\
\hline Mean age in years $( \pm S D)$ & $39 \pm 10.2$ \\
\hline Mean height in inches $( \pm S D)$ & $64 \pm 2.1$ \\
\hline Mean weight in lbs (ISD) & $141 \pm 23.8$ \\
\hline Mean number of sessions ( \pm SD) & $6.4 \pm 1.3$ \\
\hline Mean number of days between sessions ( \pm SD) & $9.0 \pm 2.5$ \\
\hline
\end{tabular}

NOTE. Values represent mean \pm SD or percent $(\%)$.

tension, relaxation, POMS) were averaged across sessions within subjects and compared with the Wilcoxin signed rank test. All data are presented as mean \pm standard deviation (SD) unless otherwise specified. Data analyses were carried out using SPSS (Release 8.0.0; SPSS Inc, Chicago, IL) for Windows. $P<.05$ is considered statistically significant.

\section{RESULTS}

\section{Demographic and Baseline Clinical Variables}

Table 1 shows the demographic and baseline clinical data for the study participants. The mean age of the sample was 39 years (range, 24 to 59 years), all were women, and $83 \%$ were registered nurses working on an infectious disease unit. Only one participant did not engage in regular exercise; the remainder exercised two or more times per

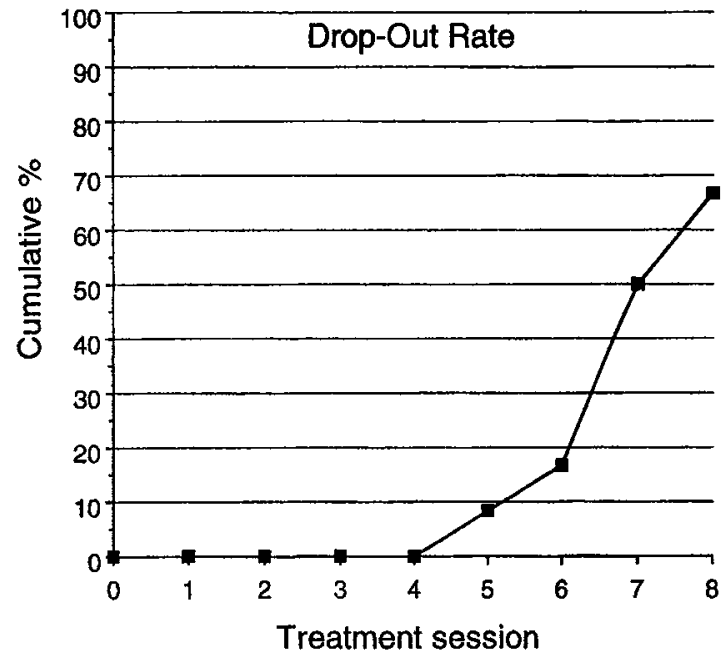

Fig 1. Cumulative drop-out rate across sessions for the 12 participants. 
Table 2. Mean \pm SD Visual Analogue Scale Pain Intensity. Tension Levels, and Relaxation Scores Before and After Massage Averaged Across all Sessions

\begin{tabular}{lcc}
\hline Variable & Premassage & Postmassage \\
\hline Pain & $1.72 \pm 0.55^{*}$ & $0.35 \pm 0.30$ \\
Tension & $3.12 \pm 1.11^{*}$ & $0.43 \pm 0.28$ \\
Relaxation & $4.65 \pm 0.94^{*}$ & $7.82 \pm 0.68$ \\
\hline
\end{tabular}

${ }^{*} P<.01$ for premassage versus postmassage comparison by Wilcoxin test.

week. Nine of the 12 participants reported tension headaches on a daily or weekly basis.

\section{Drop-Out Rates and Data on Attendance}

The mean number of massage sessions completed was 6.4 (range, 4 to 8 sessions). The mean number of days between sessions was 9 (range, 7 to 16 days). All 12 participants attended at least four sessions; thereafter, attendance rates decreased. Thirty-three percent of the sample completed all eight sessions of massage. Figure 1 shows the drop-out rate across the eight sessions.

\section{Pain, Tension, and Relaxation}

Figure 2 shows the pain, tension, and relaxation scores before and after each of the eight sessions. Wilcoxin signed rank tests showed that pain intensity and tension levels were significantly $(P<.01)$ reduced from pretreatment to post-treatment (Table 2 ). In addition, self-reported levels of relaxation increased significantly $(P<.01)$ after massage (Table 2).

\section{POMS}

Wilcoxin signed rank tests showed a significant difference from pretreatment to post-treatment on a number of POMS subscales as shown in Table 3. These analyses indicate that mood state was significantly improved after the massage treatments.

\section{Duration of Treatment Effects}

Table 4 shows the duration of the pain-relieving, tension-relieving, and relaxation effects of the massage treatments across sessions. Approximately

Fig 2. Histograms showing mean visual analogue scores for pain intensity (top panel), tension level (middle panel), and the degree of relaxation (lower panel) before and after massage on each of the eight sessions. (See Fig 1 for number of participants at each session.)
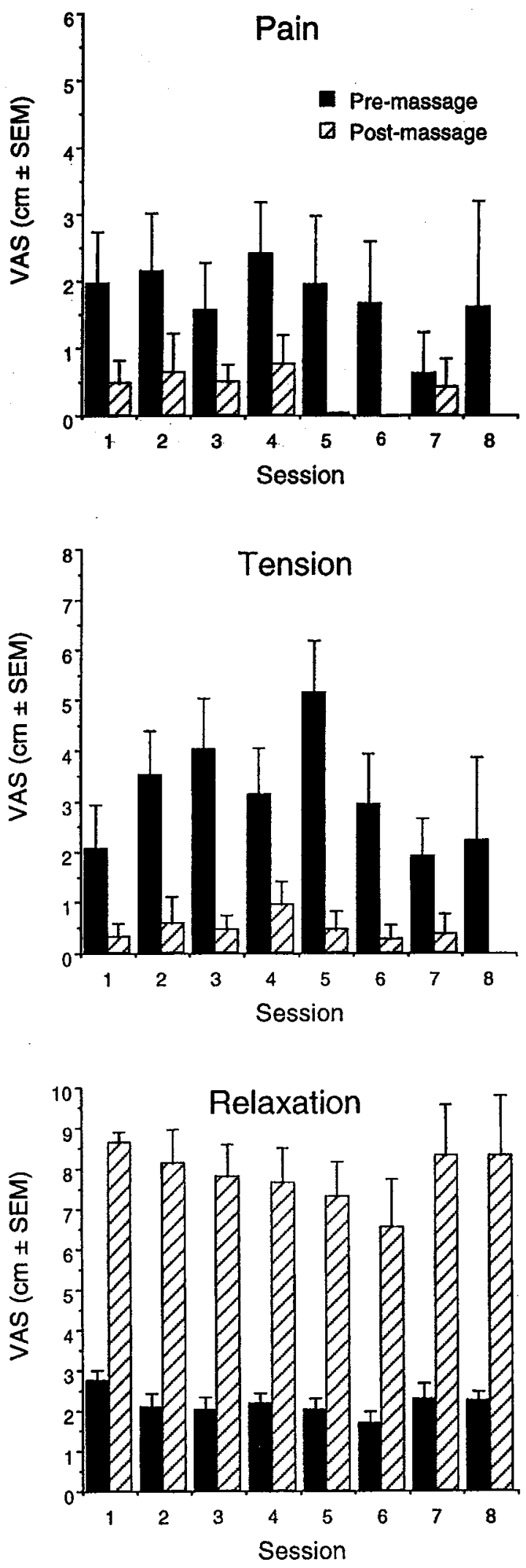
Table 3. Mean \pm SD POMS Scores Before and After Massage Averaged Across all Sessions

\begin{tabular}{lcc}
\hline \multicolumn{1}{c}{ Variable } & Premassage & Postmassage \\
\hline Tension-anxiety & $0.37 \pm 1.1^{*}$ & $-2.2 \pm 0.5$ \\
Depression-dejection & $2.0 \pm 1.4$ & $1.6 \pm 1.0$ \\
Anger-hostility & $1.9 \pm 1.5^{*}$ & $1.0 \pm 0.8$ \\
Vigor-activity & $17.4 \pm 1.8$ & $19.0 \pm 1.6$ \\
Fatigue-inertia & $4.9 \pm 2.1^{*}$ & $1.8 \pm 0.55$ \\
Confusion-bewilderment & $-1.4 \pm 0.8^{*}$ & $-2.1 \pm 0.8$ \\
Total mood score & $-9.9 \pm 6.4^{*}$ & $-18.9 \pm 3.5$ \\
\hline
\end{tabular}

$* P<.01$ for premassage versus postmassage comparison by Wilcoxin test.

$50 \%$ of the participants reported effects that persisted beyond 24 hours.

\section{DISCUSSION}

The results of this pilot study support the feasibility of an eight-session, worksite-básed, massage therapy program for pain and tension experienced by a sample of nurses working in a large teaching hospital. The results indicate that on-site massage treatment for nursing staff significantly reduced pain intensity and tension levels. In addition, after

\begin{tabular}{|c|c|}
\hline \multicolumn{2}{|l|}{ Pain Relief } \\
\hline None & $0(0 \%)$ \\
\hline Less than 1 hour & $10(14 \%)$ \\
\hline Between 1 and 24 hours & $24(33 \%)$ \\
\hline More than 24 hours & $38(53 \%)$ \\
\hline \multicolumn{2}{|l|}{ Tension Relief } \\
\hline None & $0(0 \%)$ \\
\hline Less than 1 hour & $7(10 \%)$ \\
\hline Between 1 and 24 hours & $23(33 \%)$ \\
\hline More than 24 hours & $39(57 \%)$ \\
\hline \multicolumn{2}{|l|}{ Relaxation } \\
\hline None & $0(0 \%)$ \\
\hline Less than 1 hour & $2(2 \%)$ \\
\hline Between 1 and 24 hours & $37(47 \%)$ \\
\hline More than 24 hours & $40(51 \%)$ \\
\hline
\end{tabular}

the massage treatments, participants reported significant increases in the degree of relaxation and a significant improvement in overall mood. Finally, the duration of these effects were reported to have lasted for more than a day in approximately $50 \%$ of the participants.

A limitation of the present study is the lack of a control group. It is possible that simply taking a 15-minute rest from work would have resulted in a similar degree of pain and tension reduction, and improvements in relaxation and overall mood. However, the aim of the present pilot study was to ascertain whether implementing such a program was feasible, and if so, whether the observed benefits were of sufficient magnitude to warrant conducting a randomized, controlled study of therapeutic massage. Further research is warranted to examine the efficacy of workplace-based program of massage therapy for stress and pain reduction experienced by nurses working in a large teaching hospital.

\section{the results indicate that on-site massage treatment for nursing staff significantly reduced pain intensity and tension levels. \\ ACKNOWLEDGMENTS}

This research was supported by funds from The Toronto Hospital Department of Nursing Education and Research. Dr Katz was supported by a Scholar Award from the Medical Research Council of Canada (MRC) and by MRC grant \# MT 12052. We thank Margaret Keatings for her support of the project; Diana Talley, RMT, and Andrew Dennis, RMT, for their expertise in providing the massage therapy treatments for the study participants; and Marla Jackson, BA, MHSc, for her help in data entry. We also thank Susanne Henseleit (nurse manager) for her interest in the project and the participants who took time from their busy schedules to participate in the study.

\section{REFERENCES}

1. Rest KM: Worker participation in occupational health programs: Establishing a central role. AAOHN J 44:221-225, 1996

2. Gushue J: Increasing workplace stress means occupational redicine will be a growth area. Can Med Assoc J 155:13101313, 1996

3. Ferrara-Love R: Layoff survivor sickness: How to cope when you do not lose your job. J PeriAnesth Nurs 4:236-238, 1998
4. Cady SH, Jones GE: Massage therapy as a workplace intervention for reduction of stress. Percept Mot Skills 84:157158,1997

5. Field T, Quintino $O$, Henteleff $T$, et al: Job stress reduction therapies. Altern Ther Health Med 3:54-56, 1997

6. Lewis L: Caring for the carers. Mod Midwife 5:7-10, 1995

7. Cooper $\mathrm{CL}$, Cartwright $\mathrm{S}$ : An intervention strategy for workplace stress. J Psychosom Res 43:7-16, 1997 
8. Wainapel SF, Thomas AD, Kahan BS: Use of alternative therapies by rehabilitation outpatients. Arch Phys Med Rehabil 79:1003-1005, 1998

9. Drivdahl CE, Miser WF: The use of alternative health care by a family practice population. J Am Board Fam Pract 11:193-199, 1998

10. Field $T$, Ironson G, Scafidi F, et al: Massage therapy reduces anxiety and enhances EEG pattern of alertness and math computations. Int J Neurosci 86:197-205, 1996

11. MacDonald G: Massage as a respite intervention for primary caregivers. Am J Hospice Palliat Care 15:43-47, 1998

12. Field $T$, Grizzle $N$, Scafidi $F$, et al: Massage and relaxation therapies' effects on depressed adolescent mothers. Adolescence 31:903-911, 1996

13. Huskisson $E$ : Visual analogue scales, in Melzack $R(e d)$ : Pain Measurement and Assessment. New York, NY, Raven Press, 1983, pp 33-37

14. McNair DM, Lorr M, Droppleman LF: EITS Manual for the Profile of Mood States (POMS). San Diego, CA, Educational and Industrial Testing Service, 1971 